Rochester Institute of Technology

RIT Scholar Works

Fall 12-30-2021

\title{
Evaluating Technology-Mediated Collaborative Workflows for Telehealth
}

Christopher Bondy Ph.D.

Rochester Institute of Technology

Pengcheng Shi

Rochester Institute of Technology

Pamela Grover MD

Family Medicine Specialist

Vicki Hanson

Association for Computing Machinery

Linlin Chen

Rochester Institute of Technology

See next page for additional authors

Follow this and additional works at: https://scholarworks.rit.edu/article

Part of the Databases and Information Systems Commons, Graphics and Human Computer Interfaces Commons, Operations Research, Systems Engineering and Industrial Engineering Commons, and the Telemedicine Commons

\section{Recommended Citation}

C. Bondy, P. Grover, R. Li, L. Chen, V. Hanson and P. Shi, "Evaluating Technology-Mediated Collaborative Workflows for Telehealth," in IEEE Journal of Biomedical and Health Informatics, doi: 10.1109/ JBHI.2021.3119458.

This Article is brought to you for free and open access by the Faculty \& Staff Scholarship at RIT Scholar Works. It has been accepted for inclusion in Articles by an authorized administrator of RIT Scholar Works. For more information, please contact ritscholarworks@rit.edu. 
Authors

Christopher Bondy Ph.D., Pengcheng Shi, Pamela Grover MD, Vicki Hanson, Linlin Chen, and Rui Li 


\section{Evaluating Technology-Mediated Collaborative Workflows for Telehealth}

\author{
Christopher Bondy, $\mathrm{PhD}$ \\ Golisano College of Computing \\ Rochester Institute of Technology \\ Rochester, NY, USA \\ christopher.bondy@rit.edu \\ Dr. Pamela Grover, MD, FAARM, FAAFP \\ Family Medicine Specialist \\ Penfield, NY, USA \\ drgrover@drpamgrover.com \\ Rui Li, PhD, \\ Golisano College of Computing \\ Rochester Institute of Technology \\ Rochester, NY, USA \\ rxlics@rit.edu
}

\author{
Linlin Chen, $\mathrm{PhD}$ \\ College of Science \\ Rochester Institute of Technology \\ Rochester, NY, USA \\ 1xcsma@rit.edu \\ Vicki Hanson, $\mathrm{PhD}$ \\ CEO, Association for Computing Machinery \\ Rochester, NY, USA \\ vicki.hanson@hq.acm.org \\ Pengcheng Shi, PhD \\ Golisano College of Computing \\ Rochester Institute of Technology \\ Rochester, NY, USA \\ spcast@rit.edu
}

\begin{abstract}
Goals: This paper discusses the need for a predictable method to evaluate gains and gaps of collaborative technology-mediated workflows and introduces an evaluation framework to address this need.

Methods: The Collaborative Space - Analysis Framework (CSAF), introduced in this research, is a cross-disciplinary evaluation method designed to evaluate technology-mediated collaborative workflows. The 5-step CS-AF meta-process includes: (1) currentstate workflow definition, (2) current-state (baseline) workflow assessment, (3) technology-mediated workflow development and deployment, (4) technology-mediated workflow assessment, (5) analysis and conclusions. For this research, a comprehensive, empirical study of hypertension exam workflow for telehealth was conducted using the CS-AF approach.

Results: The CS-AF systemized approach reveals critical crossdisciplinary evaluation data concerning gains and gaps of collaborative workflows when technology-mediated enhancements are characterized and compared with a baseline workflow for the goal of continuous workflow improvement.

Conclusion: The CS-AF is an effective meta-analysis process that can be adapted for use in multiple domains.

Index Terms - Collaboration, Framework, TechnologyMediated, Telehealth, Workflow.

Impact Statement - The CS-AF introduces a replicable method for evaluating the gains and gaps introduced through technology-enhancements in collaborative workflows; the CS-AF process can be adapted for use in multiple domains.
\end{abstract}

\section{INTRODUCTION}

$\mathrm{T}$ he Health Information Technology (HIT) domain is under constant transformation with its further acceleration due to the COVID-19 pandemic. Frequent introductions of new technologies leave clinicians and patients to reconcile realtime integration of collaborative workflows in order to maximize the benefits that new innovations offer. This highstakes HIT domain requires the integration of new software and hardware technologies, as well as portals, data, informatics, etc., into an ecosystem that is not always prepared for adoption.
HIT workflows are often complex and must deliver a relative advantage for a variety of patient-users with varying degrees of tech-savvy experience. Reliance on technology is becoming essential to healthcare practitioners at all levels. An overarching theme in the HIT domain is the focus on patient-centered care, which incorporates the use of new technologies (largely through software solutions) that are aimed at remoteasynchronous workflows designed for self-care and a reduction in patient hospital visits and readmissions [1]. These pressing dynamics have increased the frequency and complexity of technology-mediated collaborative workflows in telehealth, and they created stress on the entire HIT system to more efficiently evaluate and adopt new technologies [2], [3:7]. This paper discusses the system-wide need for predictable methods to evaluate the gains and gaps of technology-mediated workflows for telehealth (amongst other domains) and introduces a framework for workflow evaluation [4]. Eikey et al. conducted a comprehensive analysis of collaborative workflow in HIT over the past 25 years (surveying 943 articles); they presented a composite view of key elements that affect collaboration in HIT with their Collaborative Space Model (CSM) [5]. The CSM however, was not adapted for field use. This research builds off the theoretical approach of the CSM, expanding the view to include Attitude and Behaviors, along with Context, Technology, Process, and Outcomes, introduces a comprehensive field engagement methodology (CS-AF) meta-process, survey instrument, analysis methodology), and tests the approach with two empirical studies (graphic arts and telehealth workflows).

The Collaborative Space - Analysis Framework (CS-AF), introduced in this research, is a cross-disciplinary workflow evaluation approach designed to evaluate technology-mediated collaborative workflows [6]. Included in this research are the results of an empirical study on hypertension exam workflow using the CS-AF to evaluate a telehealth solution, versus the 
traditional (in-doctor's-office) hypertension exam workflow. The results of this research and from a prior empirical study using CS-AF for a graphic arts workflow [7] suggest wider use of the CS-AF approach for future technology-mediated workflow evaluations in telehealth and other domains with process-intense workflows.

\section{Materials AND MethodS}

The CS-AF comprises a variety of cross-disciplinary components that have been purposefully selected to enhance the view that any one single approach has on its own and to integrate the complementary attributes that each of these bestin-class models generates. The aim for the CS-AF is a generalizable, well-integrated, cross-disciplinary framework that enables a functional approach to collect and evaluate the essential data of collaborative task-oriented workflows. The CS-AF consists of five areas of investigation, a five-step implementation process, the CS-AF survey instrument, and the CS-AF data analysis procedure and summary scorecard.

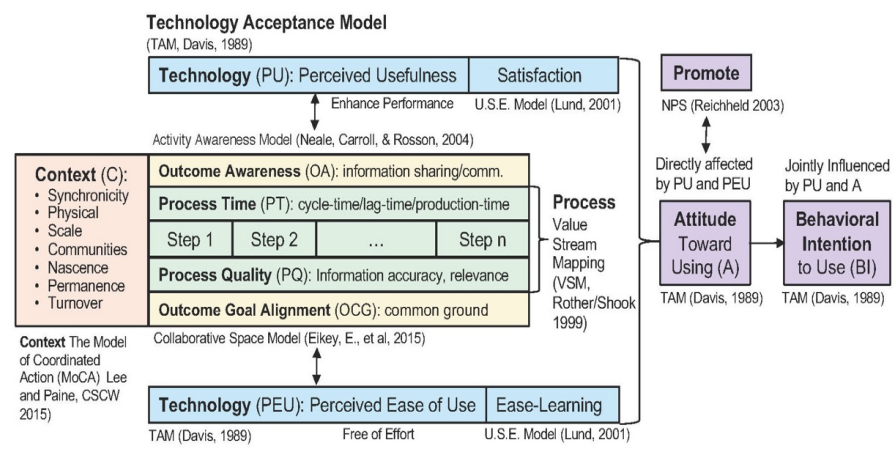

Fig. 1. Integrated cross-disciplinary components of the Collaborative SpaceAnalysis Framework (CS-AF), Bondy 2020 [6], [7], [8].

\section{A. Cross-Disciplinary Elements of the CS-AF}

Through an extensive study of related works, select components from four disciplines (Behavior Sciences, Organization Management, Industrial Engineering, and Computer Science) were integrated to formulate the CS-AF, providing a comprehensive view of a targeted workflow from five perspectives (Context, Process, Technology, Attitude and Behavioral Intention to Use, and Outcomes) (Figure 1).

The Context of the workflow is evaluated using the Model of Coordinated Action (MoCA), which enables characterization of the workflow context on seven determinants (synchronicity, distribution, scale, number of communities of practice, nascence, planned permanence, and turnover). "The seven dimensions of MoCA provide researchers, developers, and designers with a vocabulary and range of concepts that can be used to tease apart the aspects of a coordinated action that make them easy or hard to design for" [9:191].

The Process element of the CS-AF incorporates Value Stream Mapping (VSM) [10],[11], an industrial engineering hierarchical task analysis technique to uncover a quantitative view of the workflow from a time-series perspective (in each step in the workflow). VSM time-series data is complemented with further evaluation of users' awareness of information and information quality of at each stage of the workflow using techniques from Computer-Supported Cooperative Work/Human-Computer Interaction (CSCW/HCI) [12:409], [13], [14].

The CS-AF integrates aspects of the Technology Assessment Model (TAM) [15] and Lund's USE Model (Usefulness, Satisfactions, and Ease of use) [16] to evaluate the Technology aspects of the workflow (including Usefulness, Ease of Use, Satisfaction, and Ease of Learning) for each step in the workflow. Evaluating the workflow with these determinates enables a view into the specific optimization of the workflow at the task-level and complements the VSM time-series data.

The CS-AF also includes an evaluation of Attitude Toward Use and Behavioral Intent to Use the workflow, incorporating ethnographic discovery determinants from the social sciences aimed at assessing the interplay between users' feelings toward the workflow and the relative advantage they might receive [15], [17]. The Net Promoter Score (NPS) is also integrated into the CS-AF to gauge users' promotability of the workflow to others [18], [19].

The final component of the CS-AF, an evaluation of Outcomes of the workflow, focuses on goal awareness and alignment. Determinants from the CSCW/HCI (Computer Supported Cooperative Work/Human-Computer Interaction) Activity Awareness Model [13] are integrated to determine how individual users of the workflow feel others involved share mutual common ground with respect to desired outcomes at each stage of the workflow.

Through a field implementation process and survey instrument, each of the five CS-AF components are integrated for practical use.

\section{B. CS-AF Implementation Process}

Critical to the success of the CS-AF in field research (in terms of repeatability, comparative evaluation, and generalizability) is the CS-AF implementation process. Adherence to five sequential steps is essential to its successful use (Figure 2).

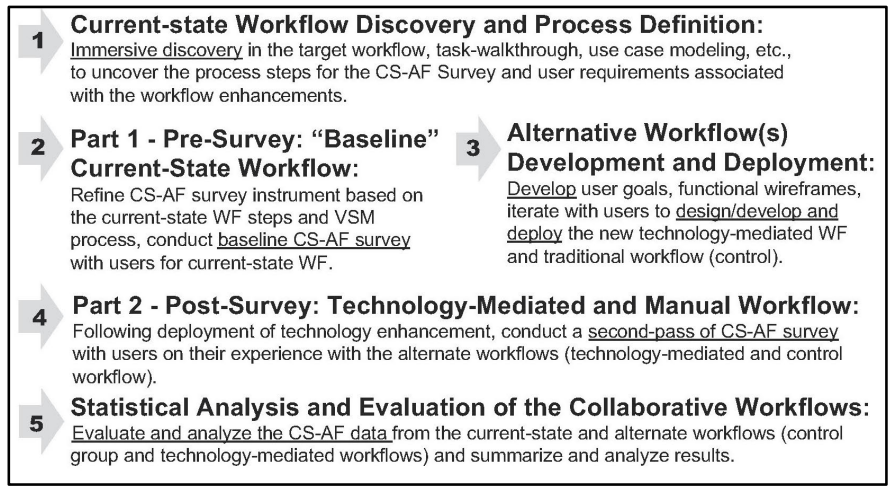

Fig. 2. CS-AF Implementation Process Reference (meta-process), [8].

Note that prior to conducting a field study, the target test environment, user profiles, and sample size, etc., for the empirical study are determined. Following the workflow audit (Step 1), the CS-AF survey instrument is refined to match the workflow steps and targeted work-task of the workflow to be evaluated. The CS-AF Implementation Process is a metaprocess intended for use in multiple domains; this process has been validated with empirical studies for two diverse domains: graphic arts and health information technology. 


\section{CS-AF Survey Instrument}

The CS-AF survey instrument incorporates 104 (7-point) Likert-scale questions, 20 quantitative time-series questions, and 15 subjective questions across the five components of the CS-AF. The CS-AF survey questions are revised for each empirical study to reflect the unique steps in the workflow; the exact same survey is used for the pre-/post-surveys. All participants were trained on the survey and associated workflow technology via remote video sessions for each group. The semi-structured CS-AF surveys were administered to test participants via an online digital survey platform with an introduction to each section and interaction for subjective questions.

For the hypertension exam workflow study, the target sample size was 50 participants -25 matched-pairs, matched on gender and 1 of 6 age bands. Of the 80 participants who were recruited, 50 were selected; all 50 participants who began the study were able to complete the study.

Using the matched-pairs approach enabled an effect size of 50 participants (type 1 error rate alpha $=0.05$, least power min. $=70 \%$, effect size $=0.5$, std. dev. $=1)$. Matched-participants $(25$ pairs) were randomly assigned to two groups (Group 1: Manual Workflow, Group 2: Technology-Mediated Workflow).

\section{Hypertension Study Test Protocol}

The hypertension exam workflow study included a baseline evaluation and survey of the current in-doctor's-office blood pressure (BP) exam by all 50 test participants. Participants were randomly divided into two groups based on their specific matched-pairs (described above). The participants in the manual workflow group (Group 1 - control group) were assigned a wrist-cuff blood pressure device. Those in the technology group (Group 2) were assigned a Bluetooth wireless bicep-cuff blood pressure device and a blood pressure app (iOS/Android) developed specifically for this study.

The clinician team involved in the study participated with patients directly during the baseline BP exam workflow and remotely through the app (BP alerts and doctor push messages) for the technology-mediated workflow, and with limited interaction for the manual wrist-cuff workflow.

All test participants attended a training session on specific test protocol and operational use of the systems they were provided. All 50 test participants conducted twice-daily BP readings per the American Heart Association's BP reading protocol [20]: two in the am (1 minute apart) and two in the pm (1 minute apart). All BP data was averaged for each day based on those four BP readings. Participants from Group 1 and 2 completed a second CS-AF survey (identical to the first), following a threeweek trial period. The CS-AF survey data was analyzed within groups and between groups. The hypertension exam workflow survey dataset comprised the analysis of 10,400 Likert-scale questions, time-series data, and 1500 subjective responses.

\section{E. CS-AF Analysis and Summary Scorecard}

CS-AF Likert-scale survey data was initially processed and analyzed using repeat measures analysis of variance ( $r$ ANOVA) to identify significant changes in group mean values for each stage of the five-step workflow [21] (Figure 3).

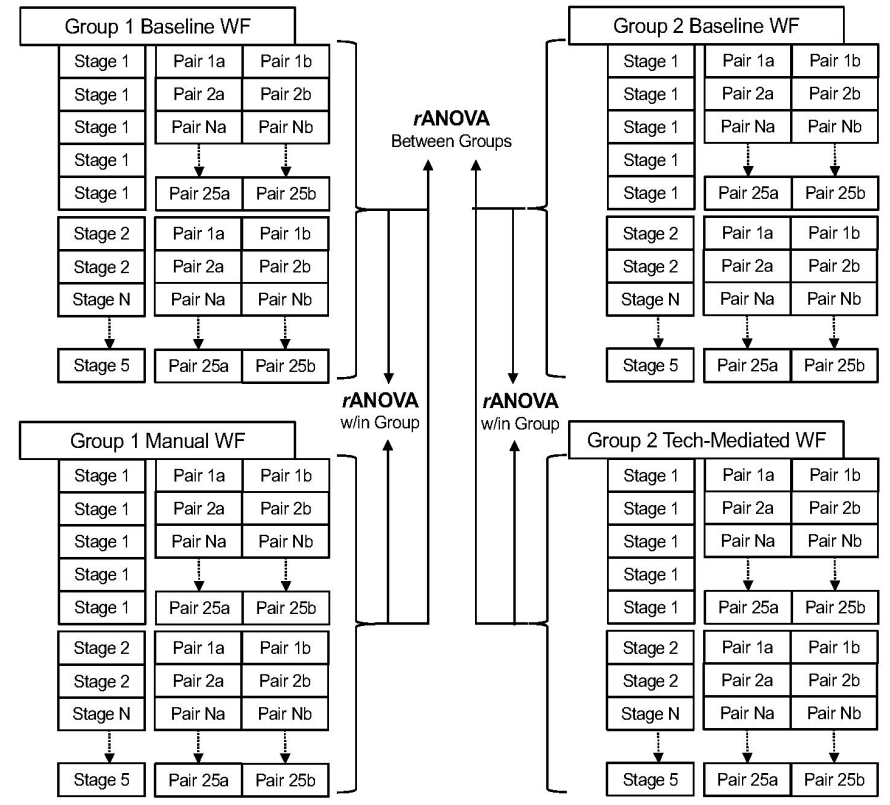

Fig. 3. rANOVA analysis of the CS-AF survey data, Hypertension exam workflow example, Bondy 2020 [8].

When p-values of $<=.05$ were evidenced, subsequent matched-pairs $t$-Tests were conducted to further identify the significant change in mean values at the specific determinant level for each Likert-scale question and specific workflow stage (Figure 4). Research shows that Likert-scale data is both interval and linear; therefore, parametric tests, such as repeat measures analysis of variance ( $r$ ANOVA) or matched pairs $t$ Test, can be used in this situation, as long certain criteria is met. Independent observations (group aggregated), homogeneity of variance, and a normal distribution are the required criteria when Likert data is to be analyzed with the use of parametric analysis. When one or more of the assumptions for the matchedpairs samples $t$-Test are not met, the nonparametric Wilcoxon Signed-Ranks Test may be run instead [21], [22], [23], [24], [25], [26:842].

The Likert data analyzed from the CS-AF surveys for the hypertension exam workflow complied with the parametric data analysis criteria and followed a normal distribution. Time-series data collected from the Process component of the CS-AF surveys was analyzed for the cycle-time, lag-time for each workflow stage, and total process time, providing quantitative time comparisons between the workflows studied.

Subjective questions were tallied, categorized into thematic clusters, and used in the final analysis to complement the statistical results of the CS-AF survey questions with thematic descriptions of user insights across the CS-AF components.

The summary results of the workflow study were tabulated in a CS-AF summary scorecard, enabling an efficient summary and visualization of the comparative results of the workflows evaluated. Group 1 (Manual Workflow, the control group) (Figure 5) and Group 2 (Technology-Mediated Workflow) (Figure 6) were compared with their baseline workflows. Group 1 and Group 2 were also compared to each other. 


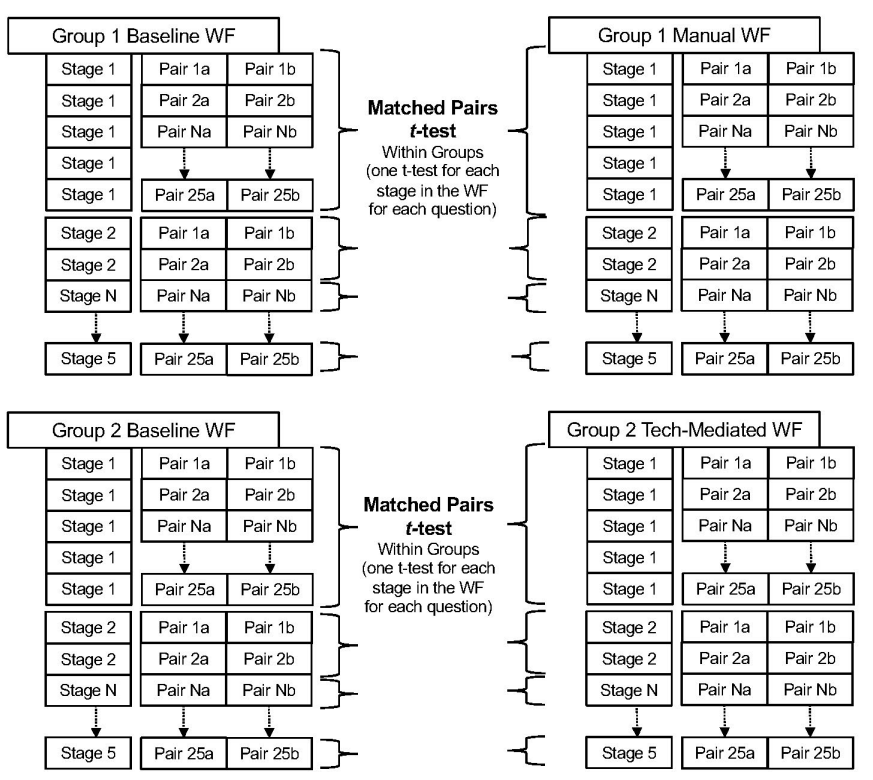

Fig. 4. Matched-pairs $t$-Test analysis of CF-AF survey data, Hypertension exam workflow example, Bondy 2020 [8].

\section{RESULTS}

CS-AF summary scorecards for within Group 1 (Figure 5) and within Group 2 (Figure 6) analysis, and analysis between Group 1 and Group 2 were performed (Figure 7). The scorecards reflect the summary data from the pre- and post-CS$\mathrm{AF}$ workflow surveys conducted for all participants and for workflows evaluated for the hypertension exam workflow study. Each respective scorecard includes summary mean values of Likert scale responses and time-series data for each of the five elements of the CS-AF and associated hypothesis H1H12. Workflows are identified as BL for baseline workflow, MN for manual workflow (Group 1), and TM for technology mediated workflow (Group 2). For each CS-AF question, the significant change in mean values $(\mathrm{p}<=.05)$ are indicated in green (pos) and red (neg); slight movement in either direction is indicated in yellow. Group 1's scorecard compares the manual (MN) workflow (wrist-cuff device) with the baseline or traditional BP exam workflow (Figure 5). Group 2's scorecard compares the technology-mediated (TM) workflow also to the baseline BP exam workflow (Figure 6). The final scorecard (Figure 7) compares Group 1 and Group 2 data.

\section{A. CS-AF Context Results}

The Context for the technology-mediated workflow compared with the baseline indicates a shift to a remote, asynchronous workflow, as hypothesized, indicative of a selfexam context (Figure 5, Figure 6). This technology-mediated workflow has transformed, from the baseline workflow, to become distributed across more locations, requiring fewer participants and communities of practice, being significantly more developing and short-term in nature, and showing slightly less turnover than in the baseline workflow. There were no surprises with these results; both groups responded as predicted to the contextual settings of the workflows.

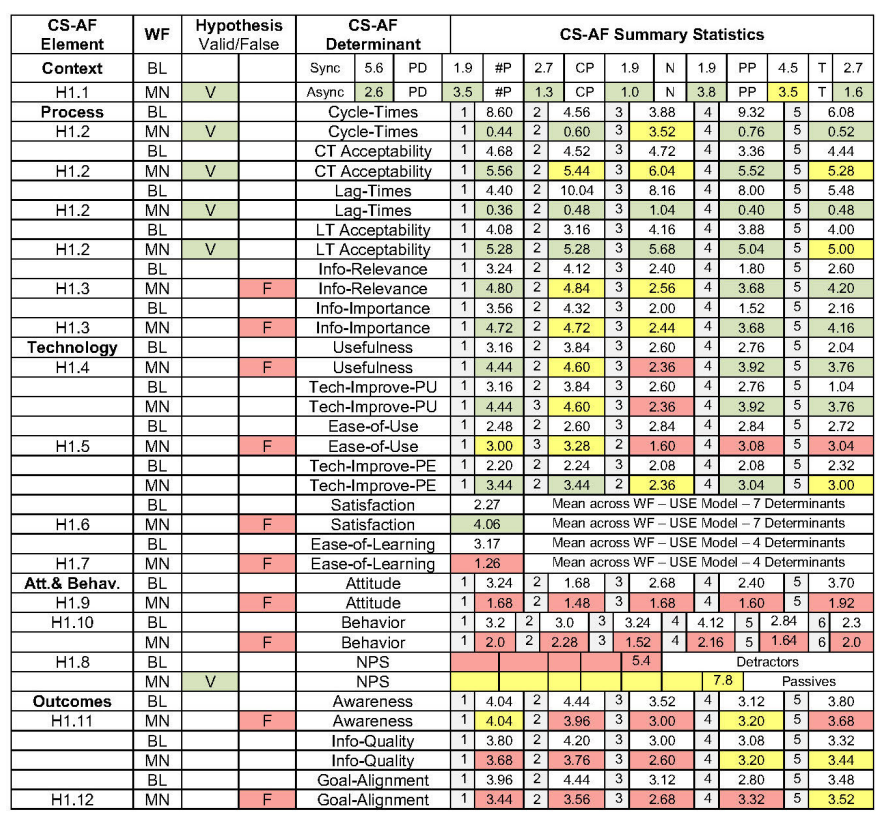

Fig. 5. CS-AF Scorecard - Hypertension Exam Workflow: Group 1 Baseline workflow summary analysis compared with the Manual (wrist-cuff) workflow summary analysis.

CS-AF Summary Scorecard Legend:

BL-Baseline Workflow, MN-Manual WF, TM-Technology-Mediated WF

Green: significant $(\mathrm{p}<=.05)$ positive, Yellow: minor positive/neg., Red: significant negative

\begin{tabular}{|c|l|}
\hline Context & $\begin{array}{l}\text { Seven MoCA determinants: Sync. vs. Async, Physical Distribution, \# } \\
\text { Participants, Communities of Practice, Nascence, Planned Permanence, } \\
\text { Turnover }\end{array}$ \\
\hline Process & Cycle time and Lag time are in .10 minutes for 5 workflow stages \\
\hline Technology & $\begin{array}{l}\text { PU and PEU, and Technology Improvement questions are calculated for 5 } \\
\text { workflow stages, Satisfaction and Ease of Learning are mean values } \\
\text { across the WF (USE). }\end{array}$ \\
\hline $\begin{array}{c}\text { Attitude \& } \\
\text { Behavior }\end{array}$ & $\begin{array}{l}\text { Attitude is assessed across the workflow for 5 determinants, Behavior is } \\
\text { assessed across the workflow for 6 determinants }\end{array}$ \\
\hline Outcomes & $\begin{array}{l}\text { Net Promoter Score (NPS) is calculated as a group mean and presented on } \\
\text { a scale from 1-10 }\end{array}$ \\
\hline & $\begin{array}{l}\text { Each question in Awareness and Goal Alignment are evaluated for the 5 } \\
\text { workflow stages }\end{array}$ \\
\hline
\end{tabular}

\section{B. CS-AF Process Results}

Analysis between Group 1 (Manual Workflow) and Group 2 (Technology-Mediated Workflow) participants indicates similar results; both the workflows proved to be successful with respect to process times (Figure 7). In fact, Group 1 was more optimized in all stages of the workflow except for Stage 3 (the BP exam). The data reflects the simplicity of the manual wristcuff workflow as more optimized for all stages, except the BP exam, since all BP data was recorded manually, in comparison to more automated results of the technology-mediated workflow.

The technology-mediated workflow scored better in the areas of information relevance and importance than Group 1, indicating the graph-plots of real-time BP data, info-graphs, alerts, and doctor messages slightly improved the quality of the information from the manual workflow (Figure 7).

\section{CS-AF Process Technology}

Technology adoption determinants rated lower than hypothesized for both workflows; however, the technologymediated solution proved slightly more "useful" than the manual solution for the first three stages of the workflow, while the results flipped for Stages 4 and 5 (Figure 6).

Participants from both groups indicated that technology 
could improve usefulness; however, the lowest rating for this determinant was Stage 3, indicating that technology could be more impactful in the front- and back-ends of the respective workflows (Figure 7).

\begin{tabular}{|c|c|c|c|c|c|c|c|c|c|c|c|c|c|c|c|c|c|c|}
\hline \multirow{2}{*}{$\begin{array}{c}\text { CS-AF } \\
\text { Element } \\
\text { Context }\end{array}$} & \multirow{2}{*}{$\begin{array}{l}\text { WF } \\
\mathrm{BL}\end{array}$} & \multicolumn{2}{|c|}{$\begin{array}{l}\text { Hypothesis } \\
\text { Valid/False }\end{array}$} & \multicolumn{3}{|c|}{$\begin{array}{c}\text { CS-AF } \\
\text { Determinant }\end{array}$} & \multicolumn{12}{|c|}{ CS-AF Summary Statistics } \\
\hline & & & & Sync & 4.6 & $\mathrm{PD}$ & 2.3 & \#P & 2. & \begin{tabular}{l|l}
2 & $\mathrm{C}$ \\
\end{tabular} & & 2.0 & $\mathrm{~N}$ & 1.6 & $\mathrm{PF}$ & & 4.3 & \begin{tabular}{l|l} 
T & 2.6 \\
\end{tabular} \\
\hline H1.1 & $\mathrm{TM}$ & V & & Async & 1.9 & PD & 4.7 & \#P & 1. & \begin{tabular}{l|l}
5 & $c$ \\
\end{tabular} & & 1.3 & $\mathrm{~N}$ & 5.0 & & $P$ & 3.8 & \begin{tabular}{l|l}
$\mathrm{T}$ & 2.3 \\
\end{tabular} \\
\hline Process & $\mathrm{BL}$ & & & \multicolumn{3}{|c|}{ Cycle-Times } & 1 & 9.92 & 2 & 4.52 & & 3 & 4.68 & 4 & & 0.5 & 5 & 3.12 \\
\hline \multirow[t]{2}{*}{$\mathrm{H} 1.2$} & $\mathrm{TM}$ & V & & \multicolumn{3}{|c|}{ Cycle-Times } & 1 & 2.28 & 2 & 1.72 & & 3 & 3.22 & 4 & & 86 & 5 & 1.72 \\
\hline & $\mathrm{BL}$ & & & CTA & ccept: & bility & 1 & 4.56 & 2 & 4.72 & & 3 & 5.32 & 4 & & 04 & 5 & 4.06 \\
\hline \multirow[t]{2}{*}{$\mathrm{H} 1.2$} & $\mathrm{TM}$ & v & & \multicolumn{3}{|c|}{ CT Acceptability } & 1 & 5.80 & 0 & 5.84 & & 3 & 6.00 & 4 & & 72 & 5 & 5.60 \\
\hline & $\mathrm{BL}$ & & & \multicolumn{3}{|c|}{ Lag-Times } & 1 & 4.88 & 2 & 11.6 & & 3 & 7.82 & 4 & & 68 & 5 & 4.48 \\
\hline \multirow[t]{2}{*}{$\mathrm{H} 1.2$} & $\mathrm{TM}$ & V & & \multicolumn{3}{|c|}{ Lag-Times } & 1 & .88 & 2 & 1.72 & & 3 & 1.12 & 4 & & 60 & 5 & 1.28 \\
\hline & $\mathrm{BL}$ & & & \multirow{2}{*}{\multicolumn{3}{|c|}{$\begin{array}{l}\text { LT Acceptability } \\
\text { LT Acceptability }\end{array}$}} & 1 & 4.08 & 2 & 3.92 & & 3 & 4.08 & 4 & & 96 & 5 & 4.60 \\
\hline \multirow[t]{2}{*}{$\mathrm{H} 1.2$} & $\mathrm{TM}$ & V & & & & & 1 & 5.88 & $\frac{2}{2}$ & 5.76 & & 3 & 5.84 & 4 & & 52 & 5 & 5.44 \\
\hline & $\mathrm{BL}$ & & & \multicolumn{3}{|c|}{ Info-Relevance } & 1 & 3.44 & 2 & 3.68 & & 3 & 2.16 & 4 & & 80 & 5 & 2.80 \\
\hline \multirow[t]{2}{*}{$\mathrm{H} 1.3$} & $\mathrm{TM}$ & & $\mathrm{F}$ & \multicolumn{3}{|c|}{ Info-Relevance } & 1 & 3.52 & 2 & 3.96 & & 3 & 2.40 & 4 & & 88 & 5 & 3.04 \\
\hline & $\mathrm{BL}$ & & & \multicolumn{3}{|c|}{ Info-Importance } & 1 & 3.64 & 2 & 4.04 & & 3 & 2.12 & 4 & & 72 & 5 & 2.68 \\
\hline $\mathrm{H} 1.3$ & $\mathrm{TM}$ & & $\mathrm{F}$ & \multicolumn{3}{|c|}{ Info-Importance } & 1 & 4.08 & 2 & 4.12 & & 3 & 2.16 & 4 & & 52 & 5 & 3.12 \\
\hline Technology & $\mathrm{BL}$ & & & & sefulne & & 1 & 3.60 & 2 & 3.44 & & 3 & 2.84 & 4 & & 68 & 5 & 3.00 \\
\hline H1.4 & TM & & $F$ & & sefulne & & 1 & 3.72 & 2 & 3.68 & & 3 & 2.20 & 4 & & 16 & 5 & 3.40 \\
\hline & $\mathrm{BL}$ & & & & nprove & & 1 & 3.60 & 2 & 3.44 & & 3 & 2.84 & 4 & & 68 & 5 & 3.00 \\
\hline & $\mathrm{TM}$ & & & & nprove & & 1 & 3.72 & 3 & 3.68 & & 3 & 2.20 & 4 & & 16 & 5 & 3.40 \\
\hline & $B L$ & & & & se-of-t & & 1 & 3.12 & 2 & 2.96 & & 3 & 3.24 & 4 & & 24 & 5 & 3.20 \\
\hline $\mathrm{H} 1.5$ & TM & & $\mathrm{F}$ & & se-of-t & & 1 & 2.96 & 3 & 3.0 & & 2 & 2.24 & 4 & & 32 & 5 & 3.44 \\
\hline & $\mathrm{BL}$ & & & & nprove & & 1 & 2.60 & 2 & 2.6 & & 3 & 2.84 & 4 & & 08 & 5 & 2.96 \\
\hline & $\mathrm{TM}$ & & & & nprove & & 1 & 3.92 & 3 & 3.9 & & 2 & 2.44 & 4 & & .00 & 5 & 3.32 \\
\hline & $\mathrm{BL}$ & & & & tisfact & & & 4.18 & & Mean & acr & sss $w$ & $\mathrm{VF}-\mathrm{US}^{\mathrm{S}}$ & $\mathrm{Mc}$ & odel - & $-7 \mathrm{Det}$ & termi & inants \\
\hline $\mathrm{H} 1.6$ & $\mathrm{TM}$ & & $F$ & & tisfact & & & 2.78 & & Mean & $\mathrm{acr}$ & sss $W$ & $\sqrt{F-U s}$ & SEMC & odel - & $-7 \mathrm{Det}$ & termi & inants \\
\hline & $\mathrm{BL}$ & & & Ease & -of-Lea & aning & & 3.40 & & Mean & acr & sss $W$ & $\mathrm{VF}-\mathrm{US}^{\mathrm{S}}$ & SEMC & odel - & $-4 \mathrm{Det}$ & termi & inants \\
\hline $\mathrm{H} 1.7$ & $\mathrm{TM}$ & & $F$ & Ease & -of-Les & aning & & 2.10 & & Mean & acr & sss $W$ & $\mathrm{VF}-\mathrm{US}$ & SEMC & odel - & $-4 \mathrm{De}$ & termi & inants \\
\hline Att.\& Behav. & $\mathrm{BL}$ & & & & Attitud & & 1 & 3.32 & 2 & 2.4 & & \begin{tabular}{l|l}
3 & \\
\end{tabular} & 3.36 & 4 & 3. & & 5 & 4.00 \\
\hline $\mathrm{H} 1.9$ & $T \mathrm{TM}$ & & $\mathrm{F}$ & & Attitud & & 1 & 2.16 & 2 & 1.8 & & 3 & 2.60 & 4 & 2 & 40 & 5 & 1.85 \\
\hline H1.10 & $\mathrm{BL}$ & & & & Sehavis & & 1 & 3.6 & & 3.92 & 3 & 3.84 & \begin{tabular}{l|l}
84 & 4 \\
\end{tabular} & 4.1 & 125 & \begin{tabular}{l|l}
5 & 2. \\
\end{tabular} & 84 & \begin{tabular}{l|l}
6 & 3.0 \\
\end{tabular} \\
\hline & $\mathrm{TM}$ & & $F$ & & Sehavi & & 1 & 2.6 & 3 & 2.0 & & 1.92 & & 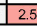 & & & 24 & \begin{tabular}{l|l}
6 & 2.6 \\
\end{tabular} \\
\hline $\begin{array}{ll}\mathrm{H} 1.8 \\
\end{array}$ & $B L$ & & & & NPS & & & & & & & 5.4 & 5.4 & & & etracts & & \\
\hline & TM & V & & & NPS & & & & & & & & & & 7.4 & & & \\
\hline Outcomes & $B L$ & & & & varene & & 1 & 4.28 & 2 & 4.6 & & 3 & 3.76 & 4 & & 60 & 5 & 3.25 \\
\hline $\mathrm{H} 1.11$ & $\mathrm{TM}$ & & $F$ & & varene & & 1 & 4.16 & 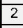 & 4.2 & & 3 & 2.76 & 4 & 2. & 92 & 5 & 3.25 \\
\hline & $\mathrm{BL}$ & & & & O-Qua & & 1 & 3.76 & 2 & 3.9 & & 3 & 3.44 & 4 & & 72 & 5 & 3.40 \\
\hline & $\mathrm{TM}$ & & & & $\mathrm{O}-\mathrm{Q}$ ua & & 1 & 3.40 & 2 & 3.4 & & 3 & 2.76 & 4 & & 80 & 5 & 3.04 \\
\hline & $\mathrm{BL}$ & & & & |-Align & ment & 1 & \begin{tabular}{|l|l}
3.60 \\
\end{tabular} & 2 & 3.8 & & 3 & 3.04 & 4 & & 56 & 5 & 3.08 \\
\hline $\mathrm{H} 1.12$ & TM & & $F$ & & |-Align & ment & 1 & \begin{tabular}{|l|l}
3.76 \\
\end{tabular} & 2 & 3.7 & & \begin{tabular}{|l|}
3 \\
\end{tabular} & 2.84 & 4 & & 00 & 5 & 3.20 \\
\hline
\end{tabular}

Fig. 6: CS-AF Scorecard - Hypertension Exam Workflow: Group 2 baseline workflow summary analysis compared with the Technology-Mediated workflow summary analysis.

Group 1 participants rated the manual workflow to be "easier to use" than did Group 2 participants on their workflow. The manual solution was reported to be an easier solution to use, compared with technology-mediated solution: however, Group 2 participants reported a higher rating for technology's ability to improve ease of use, most significantly in the front-end process for Stages 1 and 2. Both groups agreed that the hypertension exam workflow would be more beneficial with automation of the registration and appointment scheduling aspects of the workflow (Figure 7).

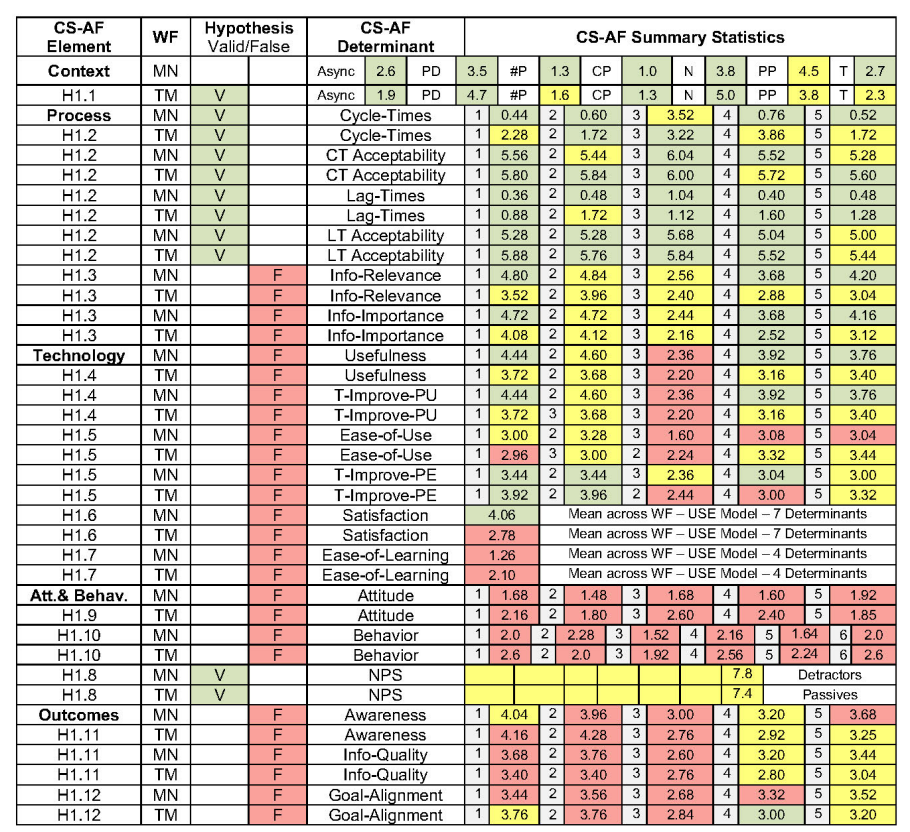

Fig. 7. CS-AF Scorecard - Hypertension Exam Workflow: Group 1 Manual (wrist-cuff) workflow summary analysis compared with the Technology-

Mediated workflow summary analysis.

Group 1 participants were overall more satisfied with the manual workflow than were Group 2 participants. Both groups found Ease of Learning for the alternative workflows to be difficult, with a surprising, slight advantage in Ease of Learning for Group 2. Low scores reported for technology adoption by both groups illustrate the burden that users carry associated with switching to technologies (Figure 7).

\section{CS-AF Attitudes \& Behavior Results}

Both groups rated determinants for Attitude and Behavior for the alternative workflows as low overall for all stages of the workflow. Group 2 scored slightly higher for all but Stage 5 for Attitude toward Using and slightly higher than Group 1 for all stages but Stage 2 for Intent to Use. The data indicates a slightly improved Attitude and Behavioral Intent of Group 2 participants to the technology-mediated workflow (Figure 7). However, it should be noted that, of all the metrics incorporated in the CS-AF, Attitude and Behavior determinates were overall the lowest scores reported. This underscores the tremendous importance of attitude and behavior on adoption in collaborative workflow and a target area for further research and development.

\section{E. CS-AF Outcomes Results}

Comparison of Outcomes between groups indicated lower participants' scores for Awareness and Information Quality from their respective baselines in Stages 1,2, and 3; and some minor improvements in Stages 4 and 5. Low scores indicate a lack of collaborative connection with clinicians for the alternative workflows (Figure 7). Participants stated that they would like more interaction and access to physician assistants (PAs) during the exam process to ask real-time questions and obtain support. With respect to Goal Alignment, Group 1 reported lower scores for the first four stages of the manual workflow and a slight increase in Stage 5 (Figure 5). Group 2 reported a slight increase in goal alignment for Stages 1, 4, and 5 , with Stage 4's increase being significant compared with the baseline (Figure 6).

Both groups reported that the problems with goal alignment are primarily in the front-end process: pre-visit, registration, and exam stages (Figure 7). This data aligns with other CS-AF data and subjective comments from participants that clinicians seem detached with respect to their specific goals in the baseline workflow. This theme extends to the alternate workflows, since being remote further amplifies the disconnect from clinicians that is already problematic. Further effort is needed in the area of goal alignment and communication for patients to be satisfied with remote telehealth and self-exams.

\section{Discussion}

A variety of valuable themes were derived from this study that highlight issues, present opportunities, and identify focus areas for further research and development.

1) Theme 1: Capture the context. The context of the workflow is an essential reference point to secure future evaluations and comparisons to technology-mediated workflow enhancements [17], [27:7]. The CS-AF approach 
proved successful in capturing details regarding the workflow that can only come from immersive engagement in the domain and workflow discovery directly with target users. Lee and Paine suggest that this approach is like a GPS for contextual setting that can provide a clean and common reference [9].

2) Theme 2: A holistic "task-focused" view is needed. This study underscores the importance of an "end-to-end" view of each workflow stage by participants and the important role of "task and technology" [28]. Adapting VSM as a structured method to uncover the specific workflow details (process steps, issues, and opportunities) proved to be instrumental in gaining the prerequisite knowledge needed to investigate, develop, and improve (alternate) workflows. To conduct workflow analysis, VSM, combined with other discovery techniques like work-task analysis, has been successfully used in a variety of industries, including telecommunications, manufacturing, information technology, and healthcare [29].

3) Theme 3: Time equals money, yet this is not the only answer. Participants across the board were pleased with the optimization of the technology-mediated workflow; however, even with marked improvement in time, participants did not feel the solution was more "useful" and their "intent to use" was actually reduced, compared with the baseline workflows. The data underscore that, although time-optimization is crucial, it is far from being the only key to a successful collaborative workflow [30]. Technology enhancements focused solely on cost effectiveness fall short of meeting the expectations of user, specifically in telehealth. The research indicates that certain "low-tech" aspects of the workflow are critical to successful adoption. Investing equal time in training, support, and collaborative dialog with users in the workflow is as important as is providing a technology solution that saves time [31].

4) Theme 4: Technology is not a substitute for 1:1 communication. The CS-AF data showed a large gap in the expectations of participants' communication with clinicians during the telehealth experience. Technology alone is not the solution to better information quality [31], [33:9]. Complicated workflows such as those studied (in the graphic arts and telehealth domains) require intermediaries to bring the transition to the new workflow. Habituation did occur for the new workflow within the three-week trial time; however, habituation is not the same as acceptance. Users were able to understand and use the new workflow effectively, yet they were not as happy with the alternative workflow because they felt that they were isolated. Coco et al. suggest that, in the case of telehealth workflow, clinicians need to be accessible to users for real-time support versus the users solely relying on the app to communicate with the doctor [31]. Technologymediated improvements need to incorporate human support in the transition time and on an ongoing basis.

5) Theme 5: Technology that is easy to use is not always adopted. The research indicates that research and developers should focus on "perceived usefulness" to help patients find the practical integration path for technology-mediated solutions into their individual health management plan. A focus on a holistic solution that improves "quality of life" versus simply providing a solution with an "easy to use" interface is required [32:7]. Breakthrough advancements in telehealth workflow will come from solutions that incorporate a complete experience, including technology, support, and integrated interaction with clinicians.

6) Theme 6: Relative advantage drives attitude and behavior to adopt. The data reveals that participants were not convinced the alternate workflows provided enough relative advantage to deem them "useful" enough to shift beliefs. [33:2]. The CS-AF incorporates "attitude and belief" metrics which prove to be critical determinants in technology adoption-specifically, in the case of elderly users who are perfectly content with traditional face-to-face workflows and who may be most impacted by remote asynchronous workflows. Elderly users are more resistant to change that they deem not as useful as the current-state workflow [34:73]. Further research is needed to understand the dynamics of helping users internalize the advantages of the technology-mediated workflow; this is a crucial aspect of technology adoption in telehealth, [35], [36].

7) Theme 7: Goal alignment requires group alignment. As large populations shift to telehealth, goal alignment determinants such as "awareness" and "common ground" may be easier in a face-to-face setting and might be overlooked in remote asynchronous telehealth workflows; "awareness" is not as natural, and breaksdowns occur in technology-mediated telehealth workflows" [36:269]. Technology-mediated telehealth solutions can also disrupt the traditional approach healthcare providers have toward establishing common ground, or shared goals, amongst their patients [38]. Telehealth solutions need to incorporate a balanced integration between data visualizations and real-time clinician support for users to migrate to telehealth workflows with the same confidence and awareness as in the traditional in-person workflows. Further research is needed to evaluate gaps identified in this study, such as behavior and attitude, with iterative design to determine the level of collaborate support necessary by the clinician team to positively influence adoption.

Dramatic change is on the horizon for healthcare and specifically for the advancement of streamlined telehealth solutions. This research and specific results from the CS-AF identified a number themes and possible solutions discussed above. The research also establishes a foundation for future research in healthcare domain that might address issues uncovered through the analysis aimed at advancement of telehealth solutions. Of specific interest are technology adoption of the elderly, accessibility, clinician support integration, and the overall telehealth experience. For successful advancement of collaborative technology-mediated telehealth workflows, a careful alignment of patients, clinicians, and researchers and developers is needed; this research identifies opportunities for advancement within these three demographics.

Patients' Perspective. Patients play a critical role in technology adoption in the telehealth ecosystem, which begins with a commitment to a personal healthcare and wellness plan 
[32:9], and includes a technology-adoption mind set [39]. Patients that meet these two criteria (personal health plan and technology mind set) would be the best candidates (i.e., users) to recruit for new technology-mediated solutions in telehealth. This research indicated that patients with these specific attributes will tend to habituate quicker and realize the relative advantage because their attitudes and behavior are more aligned with change and the acceptance of a new and different workflow [8].

Practitioners' View. Practitioners need to understand that technologies are moving at a faster rate than the medical system's ability to incorporate new capability into their traditional operations. Barrett et al. state, "Advances in technological developments significantly outpace the ability of care systems to reform themselves in a way that can provide the enabling platform necessary for wider deployment of telecare" [40]. For the provider-clinician community to be successful with telehealth, it must be viewed as an entire new implementation paradigm that is complementary with on-site care system, yet with an entirely different set of objectives, leadership, and sponsorship. This research indicates that practitioners who approach telehealth with the same mind set as they do in traditional on-site context will certainty have difficulty with telehealth technology implementations and adoption. Practitioners incorporating telehealth services must learn to redefine the context of a "patient" and the support mechanisms that will empower patients to be successful in their remote and asynchronous environments. For many, the telehealth experience is completely new and comes with a variety of apprehensions [8].

Sanders et al.'s research on barriers to participation adoption found that some telehealth patients expressed concern with being "dependent" on technology [41]. Greenhalgh et al. reported findings that telecare users had concerns about security and that there was a "perception of surveillance" [35]. Practitioners will need to understand that many of telehealth users are elderly and may have sight, hearing, and dexterity issues-amongst the typical anxiety concerns evidenced in this demographic's perception of new technology [32], [42]. Clinicians will need to establish new teams, including remotecare facilitators, project managers, and technical support specialists who are properly trained and assigned to the charter of telehealth delivery [8], [43].

Research and Developers' Perspective. Research and developers of telehealth technology can benefit from this research by shifting attention to the functional use of the technology in the field with real patients through iterative agile development involving lead-users. Since the telehealth ecosystem is just now formulating, real insight into the unmet needs of patient will be found by working directly with patients who have an interest in adopting telehealth; they can be spokespeople for their community needs [8], [29], [44].

Research and developers need to comprehend the findings in this study associated with the subtle migration of non-adopters to adopters and realize that the primary motivator is a relative advantage that triggers attitude towards use and behavioral intent to use, which feeds perceived usefulness of the technology-mediated solution for new telehealth users [33], [35], [45]. Further research and iterative design are needed to comprehend this adoption sequence. Iterative learnings can be applied to develop more holistic solutions that incorporate the needed services components that make the interpretation of relative advantage and immediate usefulness the core of the initial experience and onboarding process of new telehealth users [8].

Researchers and developers will also need to explore the technology's future space and contemplate new systems design platforms that integrate a variety of telehealth solutions into a common patient dashboard, so that patients can quickly habituate with a user experience paradigm. This approach will allow patients to gain additional relative advantage by adding in additional telehealth capability into a familiar framework that they are already comfortable with [8], [46], [47], [48].

Researchers and developers will also need to explore new ways to collaborate with the practitioner community during each stage in the product development lifecycle. This approach will facilitate better integration with providers and more targeted solutions that address the real concerns of users and practitioners. Researchers Yen and Bakken advocate an extended development lifecycle with emphasis on the front-end part of the process and iterative in nature with lead-users. [46], [48]. The telehealth research/development community is not as established as are other sectors, such as consumer electronics and business software solutions. Researcher and developers in telehealth need to investigate best practices in more mature sectors and incorporate those development lifecycle practices into their standard operating procedures to ensure predictability [8].

\section{CONCLUSION}

Development and integration of technology for collaborative workflows in telehealth introduce many variables that are of great concern to companies, organization, and individuals. These variables include the costs of development, switching costs associated with migrating from the current workflow to technology-enhanced workflows, and details of how the technology-mediated workflow functions, compared to the current workflow. There is however, no consistent approach to evaluate and compare an existing workflow with the technology-mediated workflow enhancements in a manner that identifies the improvements (gains) and barriers (gaps) in replicable manner.

The three primary objectives of this research are targeted to address this problem: (1) investigation of cross-disciplinary related works to determine a functional and comprehensive approach to evaluate collaborative technology-mediated workflows, (2) develop a field implementation and evaluation methodology, and test the framework through diverse empirical studies, and (3) formalize the approach into a replicable and generalizable framework that can be transformed for use in multiple domains. Future work should be aimed and additional empirical studies using the CS-AF in the telehealth domain, as well as in other domains.

\section{ACKNOWLEDGMENT}

Prof. Christopher Bondy, PhD, acknowledges the following researchers for their contribution towards this research: Dr. Pamela Grover, for her participation in the hypertension exam workflow study and for the enlistment and participation of her 
patients in the study. Prof. LinLin Chen, PhD, RIT College of Science, for statistical methods contributions. Prof. Rui Li, $\mathrm{PhD}$, RIT Golisano College of Computing and Information Sciences for insights into model design. Vicki Hanson, $\mathrm{PhD}$, CEO, Association for Computing Machinery, for empirical study test protocols and technology adoption in elderly. Pengcheng Shi, PhD, RIT, Associate Dean for Research/PhD Director for his overarching research advice and guidance throughout this research.

\section{REFERENCES}

[1] K. Winbladh, H. Ziv, and D.J. Richardson, "Evolving requirements in patient-centered software," in Proceedings of the 3rd Workshop on Software Engineering in Health Care, Honolulu, HI, May 22-23, 2011.

[2] E. Dorsey and E. Topol, "State of telehealth," The New England Journal of Medicine, vol. 375, no. 2, pp. 154-61, 2016.

[3] D. Barrett, J. Thorpe, and N. Goodwin, "Examining perspectives on telecare: Factors influencing adoption, implementation, and usage," Smart Homecare Technology and TeleHealth, vol. 3, pp. 1-8, 2015.

[4] M. L. Jacobs, J. Clawson, and E. D. Mynatt, "Comparing health information sharing preferences of cancer patients, doctors, and navigators," in Proceedings of the 18th ACM Conference on Computer Supported Cooperative Work and Social Computing, 2015, pp. 808-818.

[5] (CSM) E. Eikey, M. Reddy, and C. Kuziemsky, "Examining the role of collaboration in studies of health information technologies in biomedical informatics: A systematic review of 25 years of research," Journal of Biomedical Informatics, vol. 57, pp. 263-277, 2015, http://dx.doi.org/10.1016/j.jbi.2015.08.006.

[6] (TAGA) Bondy, Christopher, "Exploring the association between current state and future state technology-mediated collaborative workflow: Graphic communications workflow technical association of the graphic arts," presented at the Annual TAGA Convention, Washington, D.C., March 19, 2018

[7] (early CS-AF - IEEE) C. Bondy, "Understanding critical barriers that impact collaborative doctor-patient workflow," presented at the 2017 IEEE International Conference on Biomedical and Health Informatics, Orlando, Florida, 2017.

[8] Bondy, Christopher, "A Framework for Evaluating TechnologyMediated Collaborative Workflow" (2021). Thesis. Rochester Institute of Technology. Accessed from:

https://scholarworks.rit.edu/cgi/viewcontent.cgi?article=11854\&context $=$ theses

[9] C.P. Lee and D. Paine, "From the matrix to a model of coordinated action (MoCA)," in Proceedings of the 18th ACM Conference on Computer Supported Cooperative Work and Social Computing, 2015.

[10] M. Khurum, "Extending value stream mapping through waste definition beyond customer perspective," Journal of Software: Evolution and Process, vol. 26, pp. 1074-1105, March 2014, doi:10.1002/smr.1647

[11] S. Haizatul and R. Ramian, "Patient process flow improvement: Value stream mapping," Journal of Management Research, vol. 7, no. 2, Feb. 2015.

[12] S.K. Card, T.P. Moran, and A. Newell. "The keystroke-level model for user performance time with interactive systems," Communications of the ACM, vol. 23, no. 7 pp. 396-410, 1980, doi:10.1145.358886.358895

[13] D. C. Neale, L. Hobby, J. M. Carroll, and M. B. Rosson, "A laboratory method for studying activity awareness," in Proceedings of 3rd Nordic Conference on Human-Computer Interaction, no. 1, pp. 313-322, 2004.

[14] D.C. Neale, J.M. Carroll, and M.B. Rosson, "Evaluating computersupported cooperative work: Models and frameworks," Proceedings of the ACM Conference on Computer Supported Cooperative Work and Social Computing, pp. 112-121, 2004.

[15] F. Davis, "Perceived usefulness, perceived ease of use, and user acceptance of information technology," MIS Quarterly, vol. 13, no. 3, p. 319,1989

[16] A.M. Lund, "Measuring usability with the USE questionnaire," Usability Interface, vol. 8, no. 2, pp. 3-6, 2001.

[17] I. Ajzen and M. Fishbein. "Attitude-Behavior relations: A theoretical analysis and review of empirical research," Psychological Bulletin, vol. 84, no. 5, pp. 888-918, 1977.
[18] F.F. Reichheld, "The microeconomics of customer relationships," MIT Sloan Management Review, vol. 47, pp. 73-78, 2006.

[19] F.F. Reichheld, The Ultimate Question: Driving Good Profits and True Growth. Boston: Harvard Business School Press, 2006.

[20] Whelton PK, Carey RM, Aronow WS. Casey DE Jr. Collins KJ, Dennison Himmelfarb C. et al. 2017

ACC/AHA/AAPA/ABC/ACPM/AGS/APhA/ASH/ASPC/NMNPCNA guideline for the prevention, detection. evaluation. and management of high blood pressure in adults: a report of the American College of Cardiology/American Heart Association Task Force on Clinical Practice Guidelines. J Am Coll Cardiol. 2018:71(19).

[21] Witte, R. S., \& Witte, J. S., Statistics (9th ed.) Hoboken, NJ: Wiley, 2010.

[22] F. Wilcoxon, "Individual comparisons by ranking methods," Biometrics Bulletin, vol. 1, no. 6, pp. 80-83, Dec, 1945.

[23] G. Divine, H. J. Norton, R. Hunt, MD, and J. Dienemann, "Review of analysis and sample size calculation considerations for Wilcoxon Tests, Anesthesia and Analgesia, vol. 117, iss. 3, pp. 699-710, 2013.

[24] G.E. Meek, C. Ozgur, and K. Dunning, "Comparison of the $t$ vs. Wilcoxon Signed-Rank Test for Likert scale data and small samples," Journal of Modern Applied Statistical Methods, vol. 6, iss. 1, art. 10, 2007, doi: $10.22237 / \mathrm{jmasm} / 1177992540$.

[25] A.J. Vickers, "Comparison of an ordinal and a continuous outcome measure of muscle soreness," International Journal of Technology Assessment in Healthcare. vol. 15, no. 4, 1999, pp. 709-716, 1999.

[26] S. E. Harpe, "How to analyze Likert and other rating scale data," Currents in Pharmacy Teaching and Learning, vol. 7, iss. 6, pp. 836-850, 2015, doi:10.1016/j.cptl.2015.08.001

[27] D. Barrett, J. Thorpe, and N. Goodwin, "Examining perspectives on telecare: Factors influencing adoption, implementation, and usage," Smart Homecare Technology and TeleHealth, vol. 3, pp. 1-8, 2014, doi10.2147/SHHT.S53770

[28] S.A. Brown, A.R. Dennis, and V. Venkatesh, "Predicting collaboration technology use: Integrating technology adoption and collaboration research," Journal of Management Information Systems, vol. 27, no. 2, pp. 9-53, 201 org/stable297801700, https://jstor.

[29] (212) Harvard Business Review, vol. 79, no. 5, pp. 3-9, Sept.-Oct. 1999, https://hbr.org/1999/09/creating-breakthroughs-at-3m

[30] W.H. Woertman, G. Van De Wetering, and E.M.M. Adang, "Costeffectiveness on a local level: Whether and when to adopt a new technology," Medical Decision Making, vol. 334, iss. 3, pp. 379-386, 2014, doi:10.1177/0272989X13497995

[31] L. Coco, A. Davidson, and N. Marrone, "The role of patient-site facilitators in tele-audiology: A scoping review," American Journal of Audiology, vol. 29, no. 3S, pp. 661-675, 2020doi:10.1044/2020_AJA19 00070

[32] J.-M. Tsai, M.-J., Cheng, H.-H. Tasi, S-W. Hung, and Y.-L. Chen, "Acceptance and resistance of telehealth: The perspective of dual-factor concepts in technology adoption," International Journal of Information Management, vol. 49, pp. 34-44, 2019, doi:10.1016/j.ijnfomgt.2019.03.003

[33] P. Zanaboni and R. Wootton, "Adoption of telemedicine: from pilot stage to routine delivery," BMC Medical Informatics and Decision Making, vol. 12, art. 1, 2012 http://www.biomedicalcentral.com/1472-6947/12/1

[34] Bran Knowles and Vicki L. Hanson. 2018. The wisdom of older technology (non)users. Commun. ACM 61, 3 (March 2018), 72-77. DOI:https://doi-org.ezproxy.rit.edu/10.1145/3179995

[35] T. Greenhalgh, G. Robert, F. Macfarlane, P. Bate, and O. Kyriakidou, "Diffusion of innovations in service organizations: Systematic review and recommendations, Milbank Quarterly, vol. 82, no. 4, pp. 581-629, 2004.

[36] S.D. Scott, R.C. Plotnikoff, N. Karunamuni, R. Bize, and W. Rodgers, "Factors influencing the adoption of an innovation: An examination of the uptake of the Canadian Heart Health Kit (HHK)," Implementation Science, vol. 3, no. 1, p. 41.

[37] M.C. Reddy, M.M. Shabot, and E. Bradner, "Evaluating collaborative features of critical care systems: A methodological study of information technology in surgical intensive care units," Journal of Biomedical Informatics, vol. 41, iss. 3, pp. 479-487, 2008

[38] C.R. Weir, K.W. Hammond, P.J. Embi, E.N. Efthimiadis, S.M. Thielke, and A.N. Hedeen, "An exploration of the impact of computerized patient documentation on clinical collaboration," International Journal of Medical Informatics, vol. 80, pp. e62-e71, 2011, doi:10.1016/j.ijmedinf.2011.01.003

[39] D. Bem, D. "Self-Perception Theory," in L. Berkowitz (ed.), Advances in Experimental Social Psychology. New York: Academic Press, 1972. 
[40] D. Barrett, J.Thorpe, and N. Goodwin, "Examining perspectives on telecare: Factors influencing adoption, implementation, and usage" Smart Homecare Technology and TeleHealth, vol.3, pp.1-8, 2015, https://doi.org/10.2147/SHTT.S53770.

[41] C. Sanders, A. Rogers, Bowen R, P. Bower, S. Hirani, M. Cartwright, R. Fitzpatrick, M. Knapp, J. Barlow, J. Hendy, T. Chrysanthaki, M. Bardsley, and S. P. Newman, "Exploring barriers to participation and adoption of telehealth and telecare within the Whole System Demonstrator trial: A qualitative study" BMC Health Services Research, vol. 12 , art. no 220,2012

[42] F. Abdullah and R. Ward, "Developing a General Extended Technology Acceptance Model for E-Learning (GETAMEL) by analyzing commonly used external factors," Computers in Human Interaction, vol. 56, iss. $\mathrm{C}$, March 2016, https://doi.org/10.1016/j.chb.2015.11.036

[43] P.S. Whitten and M.S. Mackert, "Addressing telehealth's foremost barrier: Provider as initial gatekeeper," International Journal of Technology Assessment in Healthcare, vol. 21, no.4, pp. 517-521, https://www.cambridge.org/core/journals/international-journal-of-

technology-assessment-in-health-care/article/abs/addressing-telehealthsforemost-barrier-provider-as-initialgatekeeper/69929D0F97472BF711F8532C85ED9ED5

[44] S.D. Scott, R.C. Plotnikoff, N. Karunamuni, R. Bize, and W. Rodgers, "Factors influencing the adoption of an innovation: An examination of the uptake of the Canadian Heart Health Kit (HHK)," Implementation Science, vol.3, no. 41, 2008, https://link.springer.com/article/10.1186/1748-5908-3-41

[45] S. Jalil, T. Myers, I. Atkinson, and M. Soden, "Complementing a clinical trial with human-computer interaction: Patients' user experience with telehealth," JMIR Human Factors, vol. 6, no. 2, pp. e9481, 2019, https://doi.org/10.2196/humanfactors.9481.

[46] P-Y Yen and S. Bakken, "Review of health information technology usability study methodologies," Journal of the American Medical Informatics Association, vol.19, iss. 3, pp. 413-422, May 2012, https://doi.org/10.1136/amiajnl-2010-000020.

[47] E. Dorsey and E. Topol, "State of Telehealth," The New England Journal of Medicine, vol. 375, no. 2, pp. 154-61, 2016.

[48] C. Bondy, J. Rahill, and M.L. Povio, "Immersion \& iteration: Leading edge approaches for early-stage product planning," Masters' Project, Product Development, Rochester Institute of Technology, Rochester, NY, 2007. Accessed from https://scholarworks.rit.edu/theses/8277 